\title{
Field Research Experience of Medical Students: Learning and Translation from Global to Underserved US Settings
}

\author{
Fatma H. Shalan ${ }^{1}$. Shanjida Ambia ${ }^{1} \cdot$ Beatriz Martinez ${ }^{1}$ - Eric Jon ${ }^{1}$. Ugochukwu Okorafor ${ }^{1} \cdot$ Kristen Yang $^{1}$. \\ Elizabeth Yim ${ }^{1} \cdot$ Robert M. Chamberlain ${ }^{1,2} \cdot$ Nancy Sohler $^{1} \cdot$ Khadija Msami $^{3} \cdot$ Crispin Kahesa $^{3} \cdot$ Julius Mwaiselage $^{3}$. \\ Amr S. Soliman ${ }^{1}$
}

Accepted: 24 May 2021 / Published online: 1 June 2021

(c) American Association for Cancer Education 2021

\begin{abstract}
While most medical schools in the USA provide opportunities for global health experiences, global health education is not included consistently or emphasized adequately in many medical school curricula. The City University of New York Medical School (CSOM) has a mission to educate and train students who are traditionally underrepresented in medicine to practice primary care in medically underserved communities in New York. This manuscript documents the experience of the CSOM in expanding global health education by introducing a new global health cancer training program, partnering with clinicians at the Ocean Road Cancer Institute (ORCI) in Tanzania. This manuscript illustrates the following points: (1) the CSOM curriculum that focuses on community health and social medicine; (2) the process by which students learn by developing research proposals for global cancer; (3) the field research experience and lessons learned; (4) learning about cancer and medicine in a developing country; and (5) lessons learned for translation from global to domestic underserved populations. We also suggest a checklist for future students interested in pursuing global cancer education and research, and recommendations for maximizing learning and career development of students interested in global cancer research and its application to underserved populations in the USA.
\end{abstract}

Keywords Field research $\cdot$ Learning $\cdot$ Global $\cdot$ Underserved $\cdot$ Tanzania

\section{Introduction}

Education in global health is critical for medical education. Physicians in the United States of America (USA) are increasingly faced with healthcare challenges that require understanding of health and barriers to healthcare access of people from underserved groups, including migrants. [1] The world has become increasingly interconnected and globalization now affects virtually every person's life. Increased travel and flow of people, products, services, and information between and within countries and continents

Amr S. Soliman

asoliman@med.cuny.edu

1 The City University of New York School of Medicine, 160 Convent Avenue - Harris Hall 313, New York, NY 10031, USA

2 University of Texas MD Anderson Cancer Center, Houston, TX, USA

3 Ocean Road Cancer Institute, Dar es Salaam, Tanzania exert dramatic influences on health and healthcare needs and delivery that physicians must understand.[2] Understanding social theory and global health can help students and practitioners understand and interpret the nature, effects, and limitations of medical and public health interventions. [3] The worldwide impact of the COVID-19 pandemic is a demonstration of the importance of global health competencies for all physicians.

Exposure to healthcare systems outside the home country provides US medical students with opportunities to observe varieties of medical practices, healthcare resource allocations, and different approaches to medical education and learning. Training experiences in global settings contribute to medical students' increased understanding of the global burden of disease, deeper appreciation of global public health issues, and the importance of cultural sensitivity.[4] The CUNY School of Medicine (CSOM) is a mission-driven medical school that aims to increase medical education opportunities for students from racial and ethnic groups that are traditionally underrepresented in medicine 
and focuses on improving access to primary healthcare in medically underserved communities in New York City. It is a seven-year program that offers a combined BS-MD degree. The first 3 years of the school's curriculum, which is the undergraduate part, include a substantial population health component and a longitudinal clinic experience. This early population health training and clinical exposure allows students opportunities to observe how primary care providers integrate the social aspects of medicine into their practices, and provides experiences in urban healthcare settings that add to the traditional classroom undergraduate education. The four-year portion of the medical school includes practice of medicine (POM) classes in which students learn the clinical component of how to compose a physical exam, diagnose, and treat patients. Furthermore, experiential global health education outside of the USA is offered to students through a competitive summer fellowship opportunity that is sponsored by Mack Lipkin, a noted internist in New York City, in which students explore healthcare systems outside of their own and engage in mentored research.

This manuscript documents a recent experiment at CSOM to introduce a structured global health cancer research training program that could expand on global health training opportunities at CSOM. Here, we describe the experiences of seven students (one in the final year, two in the third year, three in the second year, and one in the first year of medical school), who travelled to Tanzania as part of this experimental program over two summers in 2018 and 2019 to learn about healthcare in Tanzania by observing medical practices and contributing to local cancer-related research at the Ocean Road Cancer Institute (ORCI) in Dar es Salaam. The cancer-related research projects that the students worked on included transitioning to electronic health records and databases,[5] barriers to early breast cancer detection,[6] barriers to referrals for patients with cervical cancer,[7] clinical presentation of patients with breast cancer, and the epidemiologic characteristics of patients with cervical dysplasia, cancers related to human papilloma virus infections, and liver cancer. The following sections of this manuscript illustrate the experiences of students and include suggestions for continuation of this program and its dissemination to other US medical schools.

\section{Student Selection}

Students were selected in this program based on their interest in conducting research in in low- and middle-income countries (LMICs). An advisory committee composed of CSOM faculty interviewed the applicants after reviewing their GPAs, CVs, and personal statements to elicit their interest in research in LMICs. While speaking Swahili was a plus, it was not required because English is the language of medical education and medical records in Tanzania. The committee also considered the matching between the students' backgrounds and personalities with the setting and field site mentors in the research site.

\section{The Setting}

\section{US-Based Preparation: Development of Research Projects and IRB Approvals}

The research experience of the students in this program was based on the existing infrastructure from the Cancer Epidemiology Education in Special Populations (CEESP) Program, described elsewhere in this supplement. The mission of CEESP is to train public health students in cancer prevention in international and minority US populations. [8] CEESP has an established relationship with the ORCI in Tanzania where healthcare professionals serve as mentors for students. This collaborative effort relies heavily on the guidance of the physicians, nurses, and other hospital personnel. At ORCI, physicians care for hundreds of patients from all over Tanzania. This unique setting provides students with valuable learning experiences on how physicians utilize the available limited resources to provide the best care possible for all of their patients. CEESP has been a program geared towards public health graduate students. Recently, opportunities were made available to use the disseminated infrastructure for training and research of medical students in cancer epidemiology and prevention. The 7-week CSOM fellowship required extensive pre-travel preparations that introduced the seven medical students, co-authors on this manuscript, to public health research and allowed them to apply the skills they had developed through their community health and social medicine courses. To develop their research proposals, the students worked closely with CSOM faculty, the ORCI mentors, and CEESP students. The opportunity to virtually meet and discuss with the ORCI mentors greatly aided students in developing research projects appropriate for the patient population in Tanzania. Students discussed the delivery of patient care, availability of medical resources, and barriers to care, disease management, and prevention with the mentors. The students were also introduced to CEESP alumni who advised students on the research environment in Tanzania. The process of developing the research questions began with a comprehensive literature search to familiarize the students with knowledge of local gaps in the literature, social determinants of health, the Tanzanian healthcare setting, strategies for management and prevention of the disease, and local health literacy issues.

Once students developed and finalized the mini research proposals (1 page) that were approved by their CSOM mentors, they submitted applications for ethics review to both 
the host institution in Tanzania and the Institutional Review Board (IRB) of the City College of New York. This was the first time that this group of students wrote IRB applications as principal investigators. The students learned that IRB applications required thoughtful and detailed planning to ensure the safety and confidentiality of patients and their information. Details such as the collection and storage of data and the protection of patient identifiers had to be clearly defined and justified. Although the students had taken a medical ethics course and explored medical research ethics in several courses, their leadership of IRB applications and managing the IRB process with direct communication with the IRB staff allowed them to apply and better understand concepts and rules of medical and research ethnics. This process also reinforced values of self-guidance and self-learning for conducting clinical research.

\section{In-country Work: Implementation of Research Projects}

On arrival at the cancer hospital in Tanzania, the medical students had to navigate and educate themselves on the healthcare facility. The implementation of the research projects in a different cultural setting required interacting with various members of the facility from physicians to nurses, to IT staff, and registrars. These experiences were relevant in different ways to each of the students. Those students who had prospective clinical projects had the opportunity to interact with patients and clinical staff on a regular basis to assist in the implementation of the questionnaires. Input from clinical nurses on the student's research projects were valuable in improving the cultural relevance to the patients in the hospital. These students also benefited from observing the interactions between healthcare staff and patients. This was a beneficial chance for practicing clinical skills that could be applied by the students in their clinical training. However, not speaking Swahili limited the full benefit of understanding the healthcare provider-patient communication. Another learning experience for students was observing how low literacy of patients and traditional healers may have impacted stage at diagnosis of cancer and led to delayed seeking medical care. For the other students who had retrospective research projects or projects concentrating on hospital medical records, the experience was equally as unique. There were limited interactions with clinical staff for research purposes, but these students continuously interacted with IT staff, registrar, and recorded keepers. The students spent a greater deal learning about the healthcare system from the medical records of individual patients. For the students who interacted with patients for interview purposes, participant recruitment was determined by the number of patients that were available to be examined in the clinic. Conversely, those analyzing medical records were limited to the number of records found and individual ability to examine each record. Regardless of the type of project, the students benefitted equally from the perspectives of the hospital system in Tanzania, understanding the patient-physician dynamics and seeing the challenges patients face in acquiring adequate and appropriate care.

\section{Overcoming Challenges During the Research Process}

Several challenges are encountered in the process, from identifying the research topic up to writing and publishing the manuscripts. Narrowing down the research topic and planning for research is an important learning experience that is not usually the focus of medical school education. Learning to lead and coordinate multiple components of a research project and communicating with mentors and the Tanzanian team before and during research in Tanzania were other demanding tasks.

Another challenge was the relatively short 7-week period of research available during the summer. Rather than conventionally meeting with the research personnel after arriving in Tanzania during the summer, other forms of communication such as video chats, phone calls, and messaging needed to be used during the academic year prior to arriving to Tanzania. Discussing the projects with the Tanzanian mentors and collaborators prior to arriving in Tanzania was an informative, educational, and interesting experience. After arriving to Tanzania, prioritizing each research task and component was necessary to guarantee that the needed information, medical records, and questionnaires were collected and completed. Effective communication and efficient multi-tasking was key to fulfilling projects on time. Flexibility in availability was also essential for getting more cooperation of the field site mentors in Tanzania and for timely achieving the needed research data.

Electronic medical records (EMRs) were introduced to Tanzania in 2016, but paper medical records remain the standard form of record-keeping across Tanzania and sub-Saharan Africa.[5] While working with paper medical records was expected, the usual difficulties in sorting through, reading, and interpreting inconsistent or incomplete documentation were also challenging. Overcoming this challenge required additional independent learning, research, and communication with the local team.

After returning to the USA, continuing to work on the collected data for statistical analysis and writing manuscripts was also a demanding experience. Balancing the workload between finishing the research product with the demanding medial medical school courses and requirements was also another demanding experience. However, the combined research and medical school education was a good 
preparation for the future busy and challenging future clinical career with its training and practice.

In the future, organizing a standardized program where new students build upon the work of previous students while receiving mentorship and guidance from them would streamline the process. A part of this would be a checklist of items to be addressed by incoming students as they formulate the objectives and goals of their projects and help them prepare for the non-research-related issues of housing, travel, and day-to-day living while in the country. With this, students will be able to spend more time focusing on their projects, addressing issues that arise, and make their short time in the country more effective. An example of this may be found detailing points that students should prepare for before, during, and after the trip towards the end of this manuscript.

\section{CSOM/CEESP Shared Presentations and Professional Communication Skills}

After returning from Tanzania, presentations for the research product and findings were required as oral presentations at the CEESP post-summer career development workshop. This activity included preparing and delivering 20-min oral presentations about the field research projects, including background of the research project, methods, initial results, future directions, and learning experience. The presentations represented a challenging but enriching and useful learning experience. It was useful to present the experiences that the students built from scratch, reflect on work in progress, emphasize on challenges and opportunities, and also tie their research in underserved global settings to applications in underserved populations in the USA. The presentations also illustrated the inter-professional education experience with the public health students and elicited feedback from faculty and fellow students who attended the workshop for future directions, manuscript preparation, and recommendations for career planning based on the summer experiences.

\section{Collaborating with Public Health Students}

The CSOM students had the opportunity to work alongside CEESP public health graduate students. The public health students were an invaluable source of knowledge and experience, especially when they worked on individual projects from different perspectives. For example, the public health students advised the medical students on the logistics of the record-keeping system, the process of collecting data, and the organization and analysis of data. This collaborative, inter-professional education, and teamwork allowed students to learn from each other and enhanced the quality of their research experience and manuscripts.
When medical students did not work contemporaneously with public health students on the same projects from different perspectives, the medical students built on existing data from past CEESP projects and learned from CEESP alumni about effective methods for communication with the field site mentors and navigating the foreign research environment. The interactions with the public health students solidified the notion that healthcare is a collaborative and interdisciplinary field. This research process in Tanzania was made a lot easier, enjoyable, productive, and educational by working alongside public health students.

While some medical students built upon and continued from where public health students finished their research projects, the interaction with the off-campus mentors was productive in two ways. First, the medical students had to explain their projects to the local off-campus mentors in medical terms and in ways that would have more medical rather than public health relevance. Second, because all the off-campus mentors of research in Tanzania and other LMICs are physicians, the translation of the research projects and findings to medical practice was natural and more relevant to the medical students as well as the clinical mentors.

\section{Learning Experience}

This global healthcare experience helped the medical students cultivate a better understanding of the management and preventive healthcare in global settings with limited financial resources, low-tech equipment, and limited physician training. For example, students working on cervical cancer projects had noticed that although the gold standard for diagnosing cervical carcinoma in the USA is Pap smearing and HPV DNA, Tanzania did not have to access these cytology resources for a majority of its patient and atrisk populations. Similarly, students conducting research on breast cancer in Tanzania realized that breast self-exam and clinical exams are the tools for the majority of women rather than population-based expensive screening with mammography. The students also observed that the majority of cancer patients are diagnosed at advanced disease stages, and while cancer treatment is provided for free by the government, patients had to pay out of pocket for diagnosis.

Furthermore, the research opportunity in Tanzania allowed students to put into practice concepts and skills learned in their undergraduate epidemiology and social medicine courses. The students found that being able to observe and interact with the patient population they had learned about through literature reviews was a humbling and profound experience. Being physically present with the patient population brought to the forefront the human impact that their research may have and how it can help shape healthcare and public health policy to improve 
patient's health outcomes and enrich their quality of life. This field-based and hospital-based research encouraged students to think about populations and patient groups beyond isolated assignments in classroom settings. The field research experience and interaction with patients in Tanzania also showed the students that real applications of classroom concepts they learned in their epidemiology, social medicine, and research methods courses. Having had this experience, the students felt more prepared and ready to use the skills learned in class and in the field to better serve patients in underserved communities in the USA and to contribute to clinical and population health and clinical patient-oriented research.

\section{Translation to US Immigrant Populations}

While the medical students were able to conduct global health cancer research, they were also able to shadow and observe physicians, nurses, and healthcare professionals at different hospitals in Tanzania. Engaging in global health research requires a deep understanding of the country's healthcare system and ways for accessing and serving patients. Having this experience for students is extremely valuable. Students can learn from physicians in different LMICs about resourceful innovative and low-cost methods for diagnosis and treatment and can implement them into their future practices. Participating in grand rounds and shadowing residents in Tanzania showed different styles practiced in low-resource settings. Students also observed how physicians in a low-income country need to balance effective patient management, educating patients, and respecting patient beliefs and cultural background. The best example of that was dealing with cancer patients who needed pain management. While physicians had to respect patients' utilization of herbal medicine for pain management, they also provided pain medications to patients, when possible. Programs for educating herbalists for early detection of cancer and referral to cancer centers have been provided in low-income countries in Africa.9 This idea combined working with the norms and culture of patients and non-medical providers and also utilizing the medical system when possible and available. This concept has immediate translation to the USA especially for migrant populations where herbal medicine and untraditional care is preferred and practiced by patients.

\section{Learning About Medicine and Medical Education}

During the 7 weeks in Tanzania, students were able to visit four different hospitals/clinics. They were able to distinguish between care in rural versus urban areas and the paths of patient management and referrals. Students were also able to realize the limited access to care and needed cost and effort for patients in remote areas to get quality care in this large country. It was also educational to see the disparity in resources available for diagnosis, treatment, and prevention in remote rural areas compared to urban areas in the capital city of Dar es Salaam. However, these disparities are observed within the USA, too.

Regarding medical education in Tanzania, students were able to observe how Tanzanian medical students were taught. Lists of the [10] most common diseases/conditions were listed on hospital walls to aid in honing down on a diagnosis. In addition to referrals, students were able to appreciate first hand Tanzanian healthcare in HIV diagnosis and follow-up care. HIV clinics were a must in hospitals; HIV patients were followed for long periods of time after diagnosis and cared for intensely. Students were able to understand the preventative and primary care offered in each patient's care and reflected on how this emphasis on preventative care could be implemented in the USA.

\section{Recommendation for Global Health Experience Inclusion}

Incorporating global health into the medical curriculum is an important educational and learning tool for the career development of future physicians. Recognizing the global health variation of disease incidence, presentation, and survival may encourage learning about cultural competencies, communication skills, empathy, and creative tools for managing diseases in underserved populations in the USA. The global health experience provide students with rich learning experiences from different aspects and may enhance their medical education and future clinical practice for underserved populations in the USA.

The experience also helped the students to acquire new communication skills; build networks of colleagues, collaborators, and mentors; and learn how to be resourceful for problem solving and independent and team work. Various medical schools have already implemented globalized learning from the Karolinska Institute, Stockholm, to the University of Amsterdam's elective courses with international travel.10 The experiences encountered by students have surpassed the expectations and allowed for interactive learning. In addition, it was found that medical students who have participated in international trips had higher National Board of Medical Examiners Part II scores compared to those who have not participated (possibly the effect of self-selection). [11]Therefore, intertwining the global health curriculum provides future physicians the ability to view problems with multiple perspectives. Such immersive opportunities enhance clinical understanding of students, introduce them to a perspective of medicine that cannot be experienced in 
the classroom, and reinforce learning through cross-cultural comparisons and communication. Based on the experience provided in this manuscript, the following is a suggested checklist to prepare students for their research and medical experience in foreign countries.

\section{Future Directions}

In order to further expand this global health education program, a few improvements can be implemented in the future to encourage further participation and life-long critical thinking about global health. Firstly, a global health elective can be made in which medical students who had travelled during the undergraduate years would be able to go back in the M3 or M4 year and build upon their study further. Or, a global health elective could be implemented in which students would be able to translate their research and shadowing observations to underserved populations in the USA in a clinical setting. This could further translate into students graduating with a distinct global health track. Secondly, pushing towards greater funding and grants would encourage more students to apply and engage in the program as well as having these same students recommend the importance of global health to younger classes. Lastly, to encourage further interest in global health from students, global health teachings should be incorporated even more in the typical curriculum.

\section{Checklist for Students Going Overseas}

Before trip:

Develop research idea

Perform literature search on idea and identify aspect of idea you would like to focus on

Develop research plan/timeline and identify how data would be collected

Discuss with on-site and off-site mentors feasibility of project

Finalize project and research idea

Once research plan has been fully developed, begin IRB

process with institution

attain appropriate paperwork, such as CVs, certifications from personnel, proposal

schedule an appointment with someone in the IRB office

check emails attentively for needed application edits

Reach out to students or others in the field Obtain grant/scholarship to begin planning trip
Schedule flight dates and time-keep into account the time difference and your arrival

Schedule hotel reservations-contact hotel about extended stay prior to trip or investigate other local lodgings

Get travel insurance via school/university

Update US passport if necessary

Travel visas (if traveling abroad; if there are scheduled layovers, check the visa status for the country)

Obtain relevant vaccinations and proofs, and medications Learn some basic terms in the language of the country

Reflect on the preparation and expectations of visit to the country

During trip (at research site):

Update hospital/clinic staff upon arrival and schedule date to discuss beginning project and logistics

Introduce yourself in-person and locate a designated work place for you in hospital/clinic

Begin research process-consult on-site director if you may have any questions regarding project

Explore hospital/clinic get a good understanding of healthcare and patients perspectives

Ask to shadow to understand the doctor-patient relationship

Update off-site and on-site directors of your progress weekly

If obstacles or problems begin to appear, discuss with on-site and off-site directors ways project can be altered to overcome them

Ask questions regarding the culture of the country and the hospital to gain context on doctor-patient relationship

After trip:

Thank hospital staff for welcoming you and allowing research to be done in their hospital/clinic

Update progress on publication and process

Report findings to help improve healthcare of patients globally

Reflect on the experience

\section{Suggestions to Improve Future Global Collaborations}

More intensive pre-departure training would be beneficial to the medical students participating in global health research experiences. This experience was provided to the medical students in an optimal time prior to clinical rotation experience, which provided them with adequate clinical knowledge to witness and understand some of the academic material 
already completed in the program. However, although there was abundant information about the country and hospital of the visit, supplementary information can be further developed on the general nature of the host country's healthcare system, as well as specifics for the site and issues concerning travel and lodging.[12-16] Communication with students from previous years would also be beneficial in sharing insight on the site visit.

Furthermore, ideas to reciprocate the invitations by the LMIC host country might be extended by medical students. $[15,16]$ Medical students from other countries can be provided equal opportunity to conduct research in various US locations or participate in clinical immersion. In addition, staff and students from other countries can provide informational sessions to students in the USA to allow for an exchange of information. This will allow for opportunities for both parties to collaborate with students and provide international network opportunities for medical students.

\section{References}

1. Battat R, Seidman G, Chadi N, et al. Global health competencies and approaches in medical education: a literature review. BMC Medical Education. 2010;10(1). https://doi.org/10.1186/ 1472-6920-10-94.

2. Knobler S (2006) The impact of globalization on infectious disease emergence and control: exploring the consequences and opportunities: workshop summary. National Academies Press, Washington, DC

3. Farmer P (2013) Reimagining global health: an introduction. Univ. of California Press, Berkeley

4. Drain PK, Holmes KK, Skeff KM, Hall TL, Gardner P (2009) Global health training and international clinical rotations during residency: current status, needs, and opportunities. Acad Med 84(3):320-325. https://doi.org/10.1097/acm.0b013e3181970a37

5. Okoroafor U, Mwaiselage J, Calixte R, Kahesa C, Msami K, Dorn J, Soliman AS (2019) Evaluating a newly-developed system for electronic medical records in Tanzania An example of the experience in low-income countries. J Registry Manag 46(3):84-90

6. Yim ET, Rogers TB, Msami K, Calixte R, Kahesa C, Mwaiselage J, Dorn J, Soliman AS (2021) Factors related to completion of referral among women suspected of cervical cancer and dysplasia in Tanzania. Int J Gynecol Obstet 152:88-95

7. Mattick LJ, Ochs-Balcom HM, Mwaiselage J, Kahesa C, Gard ACC, Shalan F, Soliman AS. Downstaging of cervical cancer in Tanzania over a 16-year period. Cancer Causes and Control (In Press).

8. CEESP. Mission. CEESP. https://ceesp.ccny.cuny.edu/mission/. Published July 1, 2018. Accessed September 20, 2020.

9. O'Brien KS, Soliman AS, Annan K, Lartey RN, Awuah B, Merajver SD (2012) Traditional herbalists and cancer management in Kumasi. Ghana J Cancer Educ 27(3):573-579 (PMID: 22549472)

10. Bateman C, Baker T, Hoornenborg E, Ericsson U (2001) Bringing global issues to medical teaching. The Lancet 358(9292):1539-1542

11. Waddell WH, Kelley PR, Suter E, Levit EJ (1976) Effectiveness of an international health elective as measured by NBME Part II. J Med Educ 51:468-472

12. Imperato PJ, Bruno DM, Monica Sweeney M (2016) Ensuring the health safety and preparedness of U.S medical students participating in global health electives overseas. J Community Health 41(2):442-450. https://doi.org/10.1007/s10900-016-0169-7

13. Kumwenda B, Royan D, Ringsell P, Dowell J (2014) Western medical students' experiences on clinical electives in sub-Saharan Africa. Med Educ 48(6):593-603. https://doi.org/10.1111/medu. 12477

14. Lu PM, Park EE, Rabin TL et al (2018) Impact of global health electives on US medical residents: a systematic review. Ann Glob Health 84(4):692-703. https://doi.org/10.9204/aogh.2379

15. Bozinoff N, Dorman KP, Kerr D et al (2014) Toward reciprocity: host supervisor perspectives on international medical electives. Med Educ 48(4):397-404. https://doi.org/10.1111/medu.12386

16. Fotheringham EM, Craig P, Tor E (2018) International medical electives in selected African countries: a phenomenological study on host experience. Int J Med Educ 9:137-144. https://doi.org/10. 5116/ijme.5aed.682f

Publisher's Note Springer Nature remains neutral with regard to jurisdictional claims in published maps and institutional affiliations. 